\title{
Optical properties of organically functionalized silicon surfaces: Uracil-like nucleobases on $\mathrm{Si}(001)$
}

\author{
Elena Molteni, ${ }^{1,2,3}$ Giancarlo Cappellini, ${ }^{4}$ Giovanni Onida, ${ }^{1,3}$ and Guido Fratesi ${ }^{1,3}$ \\ ${ }^{1}$ Dipartimento di Fisica, Università degli Studi di Milano, via Celoria 16, 20133 Milano, Italy \\ ${ }^{2}$ OAC - Osservatorio Astronomico di Cagliari, Via della Scienza 5, 09047 Selargius (CA), Italy \\ ${ }^{3}$ European Theoretical Spectroscopy Facility (ETSF) \\ ${ }^{4}$ Department of Physics and CNR-IOM SLACS Cagliari, Università degli Studi di Cagliari, Cittadella Universitaria di Monserrato, \\ S.P. Monserrato-Sestu Km. 0.700, 09042 Monserrato (CA), Italy
}

(Received 13 October 2016; revised manuscript received 7 February 2017; published 28 February 2017)

\begin{abstract}
We predict UV reflectance anisotropy spectra (RAS) of the organically functionalized silicon (001) surface covered by pyrimidinic uracil-like nucleobases. First-principles results based on density functional theory show characteristic spectral features appearing in the UV range between 3 and $7 \mathrm{eV}$, besides the expected quench in the well-known two-minima RAS signal of clean $\mathrm{Si}(001)$. Nucleobase adsorption in the energetically favored "dimer bridge" configuration gives rise to a characteristic RAS line shape, common to thymine, uracil, and 5-fluorouracil. We trace back the origin of such spectral features by singling out RAS structures induced by relaxation and passivation effects on the $\mathrm{Si}$ surface, and those directly associated with molecular excitations. The former turn out to be the same for the three nucleobases, and are totally unaffected by molecular tilting. The sign and position of the latter RAS peaks at higher energy exhibit a moderate nucleobase dependence, and can be fully rationalized in terms of the molecular orbitals involved. The present theoretical results call for a RAS experimental study in the UV region extending up to $\simeq 6-7 \mathrm{eV}$.
\end{abstract}

DOI: 10.1103/PhysRevB.95.075437

\section{INTRODUCTION}

Organically functionalized solid surfaces have acquired a fundamental role in many fields of research, ranging from DNA sequencing $[1,2]$ to semiconductor devices with novel functionalities [3-5], also taking advantage of the great flexibility of organic chemistry in tuning the molecular electronic and optical properties [6-8]. A broad variety of molecular assemblies can form upon adsorption of organic molecules on metal, semiconductor, and insulating surfaces: a controlled growth process may lead to self-organized overstructures, with different geometries depending on the experimental conditions during growth. In this landscape, nucleobases have attracted much attention in view of their fundamental role in molecular biology and their simplicity with respect to larger biomolecules (peptides or even some amino acids) [9]. Indeed, organic/inorganic interfaces realized by chemisorption or physisorption of nucleobases such as uracil and thymine have been the subject of several experimental and theoretical studies [10-14].

We here focus on the case of $\mathrm{Si}(001)$, the technologically most important $\mathrm{Si}$ surface. For this system, monolayer/submonolayer adsorption of uracil molecules has been investigated by $a b$ initio calculations within density functional theory (DFT) within the generalized gradient approximation (GGA) $[15,16]$. In agreement with the experimental results of Lopez et al. [17], DFT results have shown that uracil molecules absorb vertically, with $\mathrm{Si}-\mathrm{O}$ and $\mathrm{Si}-\mathrm{H}$ bonds, indicating that hydrogen atoms are cleaved from the molecule, and move to saturate dangling bonds of Si surface atoms.

Some of us have recently extended ab initio DFT electronic structure calculations of Ref. [16] for uracil to the case of thymine and 5-fluorouracil, considering adsorption on the $\mathrm{Si}(001)$ surface in the same "dimer bridge" configuration [18], and including additional degrees of freedom, namely the possibility of a tilt of the molecular ring with respect to the vertical orientation. As a result, moderately tilted geometries have been shown to be favored at both the local density approximation (LDA) and GGA levels of theory. Interestingly, a tilted geometry for thymine on $\mathrm{Cu}(110)$ has also been inferred in previous works both from infrared spectra [10] and from $\mathrm{x}$-ray spectra [11], with a tilt angle of about $20^{\circ}$ emerging from the latter work.

In this paper we present a detailed study of the optical properties of $\mathrm{Si}(001)$ : $\mathrm{X}$ systems, with $\mathrm{X}=$ thymine (THY), uracil (URA), and 5-fluorouracil (5-FU), adsorbed in the "dimer bridge" configuration at a half-monolayer coverage. We analyze the effects that chemical substitutions and offvertical molecular tilting have on the optical and reflectance anisotropy spectra (RAS), identifying a characteristic line shape of RAS spectra in the near UV. This turns out to be very similar in all the considered cases with moderate differences in peak positions that can be rationalized in terms of the adsorbate orbitals.

The paper is organized as follows: first we discuss the computational methods used (Sec. II A), and the choice of adsorption configurations (Sec. II B). In Sec. III we present our results for the electronic structure, the RAS, and the general features of computed optical spectra. The link between optical absorbance and RAS, the origin of the observed features, and their dependence on the adsorbed nucleobase are discussed in Secs. IV A, IV B, and IV C, respectively. Conclusions are drawn in Sec. V.

\section{THEORETICAL AND COMPUTATIONAL METHODS}

\section{A. Computational details}

All the present calculations are performed within DFT $[19,20]$ using the exchange-correlation functional in the form by Perdew, Burke, and Ernzerhof (GGA-PBE) [21,22]. To describe the ion-electron interaction we used norm-conserving 
pseudopotentials of the Trouiller-Martins type generated via the FHI98PP [23] code. We modeled the $\mathrm{Si}(001)$ surface with a periodically repeated asymmetric slab, using a supercell containing $8 \mathrm{Si}$ layers plus a vacuum region (including adsorbed molecules) of $\sim 1.8 \mathrm{~nm}$ equivalent in thickness to $12 \mathrm{Si}$ layers, and an in-plane surface periodicity allowing for a $(4 \times 2)$ reconstruction of the top layer, where molecules are adsorbed. The Si bottom layer was saturated by hydrogen atoms. For ground-state calculations we sampled the surface Brillouin zone (SBZ) of the $(4 \times 2)$ unit cell with a $(6 \times 12)$ shifted Monkhorst-Pack mesh [24]. The kinetic energy cutoff for the plane wave expansion was set to $20 \mathrm{Ha}$.

We compute optical properties within the independentparticle random-phase approximation (IP-RPA) [25], i.e., by computing the macroscopic dielectric function of the supercell as a sum of individual valence $(v)$ to conduction $(c)$ transitions between Kohn-Sham eigenstates:

$$
\operatorname{Im} \varepsilon_{j j}(\omega)=\frac{16 \pi}{\omega^{2}} \sum_{v, c, k}\left|\left\langle\psi_{v k}\left|(\mathbf{v})_{j}\right| \psi_{c k}\right\rangle\right|^{2} \delta\left(\epsilon_{c k}-\epsilon_{v k}-\omega\right) .
$$

Here $\mathbf{v}$ is the velocity operator and $j=x, y, z$.

Following the approach of Ref. [26], many-body effects such as excitons, local fields, and self-energy corrections are neglected, relying on their partial reciprocal cancellation [27-29]. We choose to refrain from the explicit introduction of the often used rigid shift ("scissor operator") to our DFT-GGA theoretical spectra, keeping our discussion as unbiased as possible. We remark that relative energies and the dependence of the results on the adsorbed species are not influenced by such correction. A small underestimation of the experimental excitation energies, of the order of $0.5 \mathrm{eV}$, should be taken into account when comparing the absolute position of our theoretical spectra with experimental ones.

The RAS spectrum is obtained as $[30,31]$

$$
\frac{\Delta R}{R}(\omega)=\frac{4 \omega L}{c} \operatorname{Im}\left\{\frac{\Delta \varepsilon(\omega)}{\varepsilon_{\text {bulk }}(\omega)-1}\right\},
$$

where $\Delta \varepsilon=\varepsilon_{x x}-\varepsilon_{y y}, \varepsilon_{\text {bulk }}$ is the dielectric function of bulk $\mathrm{Si}, L$ the length of the simulation cell in the $z$ direction, along the surface normal, and $c$ the velocity of light. We obtain converged optical spectra in the range of interest by including empty states with energy $\epsilon_{c k}$ up to $8 \mathrm{eV}$ above the valence band maximum, and by sampling the SBZ of the $(4 \times 2)$ unit cell on a $(8 \times 16)$ shifted Monkhorst-Pack mesh [24].

To help the interpretation of the RAS spectra of the system, we also compute the absorbance of the slab model (including adsorbates) for light at normal incidence [32-34]:

$$
A_{j}(\omega)=\frac{\omega L}{c} \operatorname{Im}\left[\varepsilon_{j j}(\omega)\right]
$$

Here $j=x, y$ labels the light polarization. We remark that $A_{j}$ is independent of the thickness of the vacuum region.

For ground-state and Kohn-Sham band structure results we rely on the Quantum ESPRESSO suite [35], while in all the optical property calculations we use the Yambo code [36]. (a)

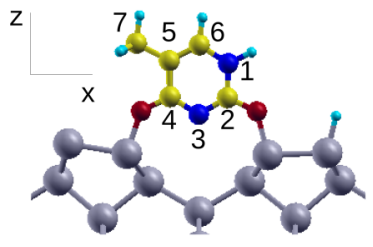

(b)

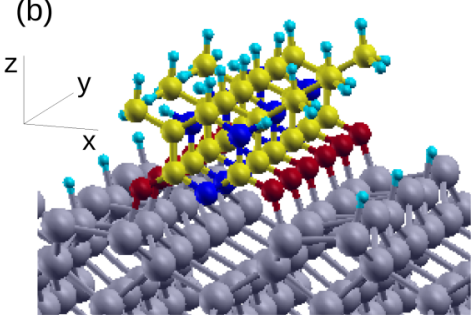

FIG. 1. Adsorption configuration for the investigated nucleobases on $\mathrm{Si}(001)$. The case of thymine is depicted: (a) side view, with standard atom labeling for the molecule; (b) perspective view. Besides Si atoms (dark gray), carbon, nitrogen, and oxygen atoms are depicted in yellow, blue, and red, respectively. Smaller cyan spheres represent hydrogen atoms. We choose as $x$ axis the direction of the Si dimers of the clean $c(4 \times 2)$ surface, so that adsorbed molecules are stacked along the $y$ direction.

\section{B. Structural details}

We consider the adsorption of thymine, uracil, and 5fluorouracil, on $\mathrm{Si}(001)$ in the "dimer bridge" configuration $[15,16,18]$, which was previously shown [15] to be among the energetically favored ones for uracil adsorption on $\mathrm{Si}(001)$ when moderate annealing temperatures are considered. This configuration has been thoroughly investigated in the literature from the structural point of view, and we briefly recall it here selecting thymine as an example [see Fig. 1(a)]. The two oxygen atoms of the pyrimidinic nucleobase are bound to two surface silicon atoms from $\mathrm{Si}$ dimers of adjacent rows. The nitrogen atom at position 3 loses its $\mathrm{H}$ which adsorbs on one of the remaining "free" Si surface atoms, saturating a dangling bond. Molecules hence form rows along the $y$ direction, i.e., perpendicularly to the Si dimer axis $(x)$ as shown in Fig. 1(b). We focus on the case of one molecule per each Si dimer along $y$ and one molecule every other Si dimer in $x$ direction, which defines a half-monolayer coverage. Facing molecules within a row are rotated by $180^{\circ}$ with respect to each other, resulting in a $(4 \times 2)$ surface reconstruction. Assuming the equivalence of the interactions of each molecule with its two nearest neighbors along the row yields a glide plane symmetry, which induces a twofold band degeneracy along the $\mathrm{K}-\mathrm{J}^{\prime}$ direction of the surface Brillouin zone $[16,18]$. Besides considering molecules adsorbed with the aromatic ring perpendicular to the Si surface, we also include the case of $\operatorname{Si}(001)$ : THY with a tilt angle of $20^{\circ}$ from the surface normal, since we previously found [18] that moderately tilted geometries are energetically favored with respect to the vertical one, both at the LDA and GGA levels of theory. A similar value of the tilt angle has been measured for THY adsorbed on $\mathrm{Cu}(110)$ [11].

\section{RESULTS}

\section{A. Electronic structure}

In Fig. 2 we show typical electronic band structure results, with the example of $\mathrm{Si}(001)$ :THY. Band states (empty/filled states at positive/negative energy values) are color-highlighted according to their projection onto the $\sigma$ and $\pi$ molecular orbitals of gas phase THY, using the procedure adopted in Ref. [37]. The right panel shows the projected density of states. 


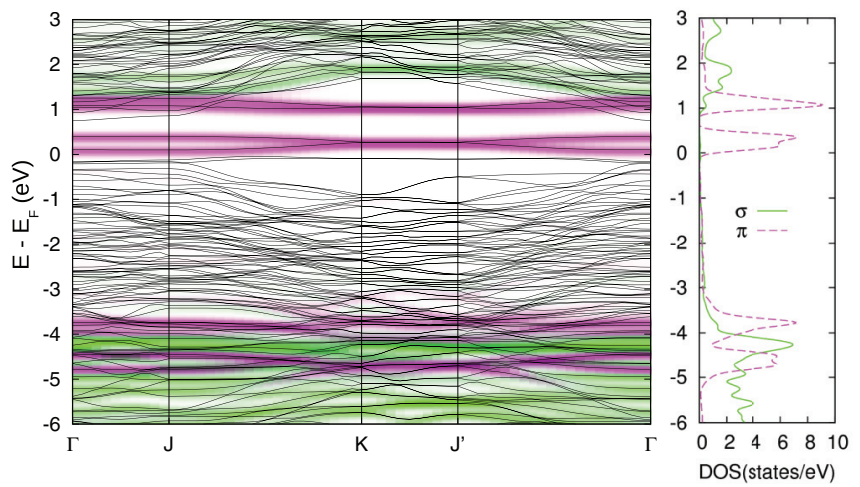

FIG. 2. Band structure of the $\mathrm{Si}(001)$ :THY system with vertically adsorbed molecules. Bands are colored according to their projection onto the molecular orbitals of gas phase THY, using magenta and green for $\pi$ and $\sigma$ states, respectively. Right panel: The $\operatorname{Si}(001)$ :THY density of states projected on the molecule, separating molecular states with $\pi$ and $\sigma$ symmetry.

The lowest unoccupied bands clearly appear as deriving from the THY LUMO and remain within the gap of the surface projected bands along the whole SBZ (the conduction band minimum being at about $0.43 \mathrm{eV}$ at $\Gamma$ ) [18]. Their slight dispersion in the $\Gamma-\mathrm{J}^{\prime}$ direction testifies to a nonvanishing intermolecular interaction within a molecular row. A similar dispersion is shown by the pair of bands originating from THY LUMO +1 orbitals, which however overlap with $\mathrm{Si}$ conduction bands near $\Gamma$ and along the $\Gamma-\mathrm{J}$ direction. In the occupied manifold, the highest occupied state of the full system (also in the gap of the surface projected bulk bands at various parts of the SBZ) has negligible contributions from molecular states, and is reminiscent of the clean $\mathrm{Si}(001)$ surface, being mainly localized on the dangling bonds of the unsaturated Si dimers [18]. In order to find THY-HOMO related bands one needs to descend about $3.7 \mathrm{eV}$ below the valence band maximum (VBM). Such bands remain quite distinguishable, although clearly overlapping with the substrate ones. Both the THY-LUMO and THY-HOMO states have a clear $\pi$ character, whereas occupied (empty) molecular $\sigma$ states are found at lower (higher) energies, as shown in Fig. 2.

\section{B. Reflectance anisotropy spectra}

Figure 3 shows the reflectance anisotropy spectra computed for the $\mathrm{Si}(001)$ :X systems. All spectra for the adsorbed systems correspond to "dimer bridge" molecular configurations, as described in Sec. II B, and are plotted for the case of vertical molecular rings. In order to analyze the effects of molecular tilting we also show the spectrum of the $\mathrm{Si}(001)$ :THY system with the thymine molecular rings set at about $20^{\circ}$ with respect to the vertical orientation. We also computed the RAS of clean $\mathrm{Si}(001)-c(4 \times 2)$ for comparison (gray circles in Fig. 3). The latter displays two characteristic negative peaks, at $\sim 1.3 \mathrm{eV}$ and at $\sim 3 \mathrm{eV}$, in agreement with previous calculations in the literature [38-40] and with experimental RAS measured on clean $\mathrm{Si}(001)$ surfaces [41], taking into account that an energy position matching the experimental one would be obtained by applying a shift of about $0.5 \mathrm{eV}$ in the calculations, as done in Refs. [38-40].

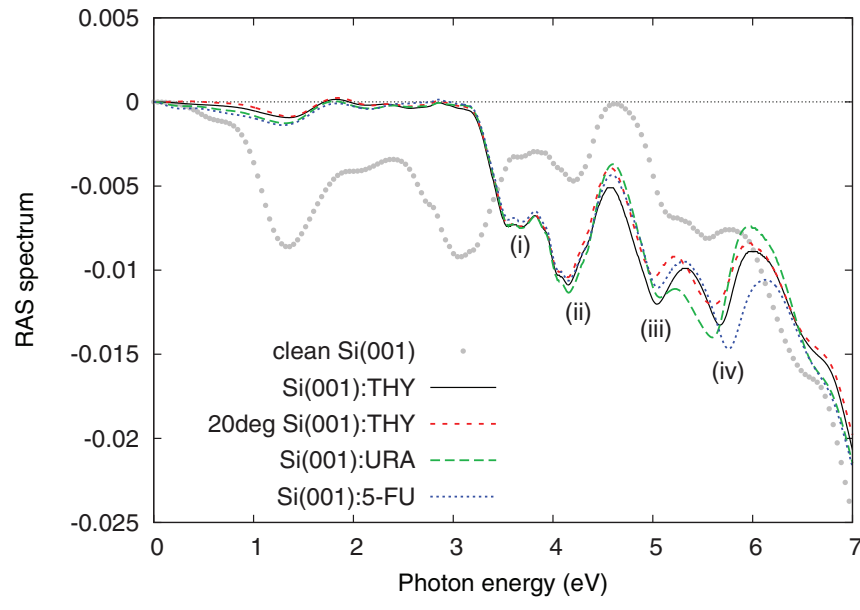

FIG. 3. RAS spectra of the vertically adsorbed $\mathrm{Si}(001)$ :THY (black solid line), Si(001):URA (green long-dashed line), and $\mathrm{Si}(001): 5-\mathrm{FU}$ (blue dots) systems. The clean $\mathrm{Si}(001)-c(4 \times 2)$ surface case is also included for comparison (gray circles). The red shortdashed line shows the RAS spectrum for $\mathrm{Si}(001)$ :THY tilted by $20^{\circ}$, illustrating the effect of molecular tilting as compared with the vertical case (black solid line).

These two peaks are known to be due to surface bands, and are strongly quenched in all our nucleobase-functionalized surfaces. The RAS spectra of all considered $\mathrm{Si}(001)$ : X systems are indeed close to zero up to $\sim 3 \mathrm{eV}$, in agreement with results for $\mathrm{Si}(001)$ :URA by Seino et al. [26]. Between 3 and $4.5 \mathrm{eV}$ the $\mathrm{Si}(001)$ :X RAS displays instead a characteristic line shape with the two negative spectral structures labeled (i) and (ii) in Fig. 3. These are remarkably independent of the molecular species adsorbed, as well as of the molecular tilt. Moving at energies above $4.5 \mathrm{eV}$, all the considered systems [i.e., $\mathrm{Si}(001)$ :THY, $\mathrm{Si}(001)$ :URA, $\mathrm{Si}(001): 5-\mathrm{FU}$, and $20^{\circ}$ tilted $\mathrm{Si}(001)$ :THY] display two more negative RAS peaks: peak (iii) at about $5 \mathrm{eV}$, and peak (iv) lying in the 5.5-6.0 eV range. While the RAS spectra for the four systems are hardly distinguishable from one another in the region below $4.5 \mathrm{eV}$, some sensitivity to molecular species and geometries appears in the strength and position of these higher energy structures. In particular, differences between THY, URA, and 5-FU can be observed mainly above $5 \mathrm{eV}$, especially concerning the energetic position of the peak (iv) that changes by $-0.08 \mathrm{eV}$ for URA and by $+0.08 \mathrm{eV}$ for 5-FU, with respect to THY. Molecular tilting results essentially in a slight reduction in anisotropy (red short-dashed line in Fig. 3). The observed changes in the RAS spectra $\left(\approx 10^{-3}\right)$ are relatively small but an order of magnitude larger than experimental capabilities [42].

\section{Optical absorbance spectra}

The observed changes in RAS spectra of the $\mathrm{Si}(001)$ surface upon nucleobase adsorption shall be understood in terms of changes in the optical absorbance spectra for light polarized along $x$ and $y$; see Eq. (3). To this aim, in Fig. 4 we compare the computed slab optical absorbances $A_{j}(\omega)$ of the clean $\mathrm{Si}(001)$ surface with those of (vertical) $\mathrm{Si}(001)$ :THY, which is taken hereafter as representative of the $\mathrm{Si}(001)$ :X 

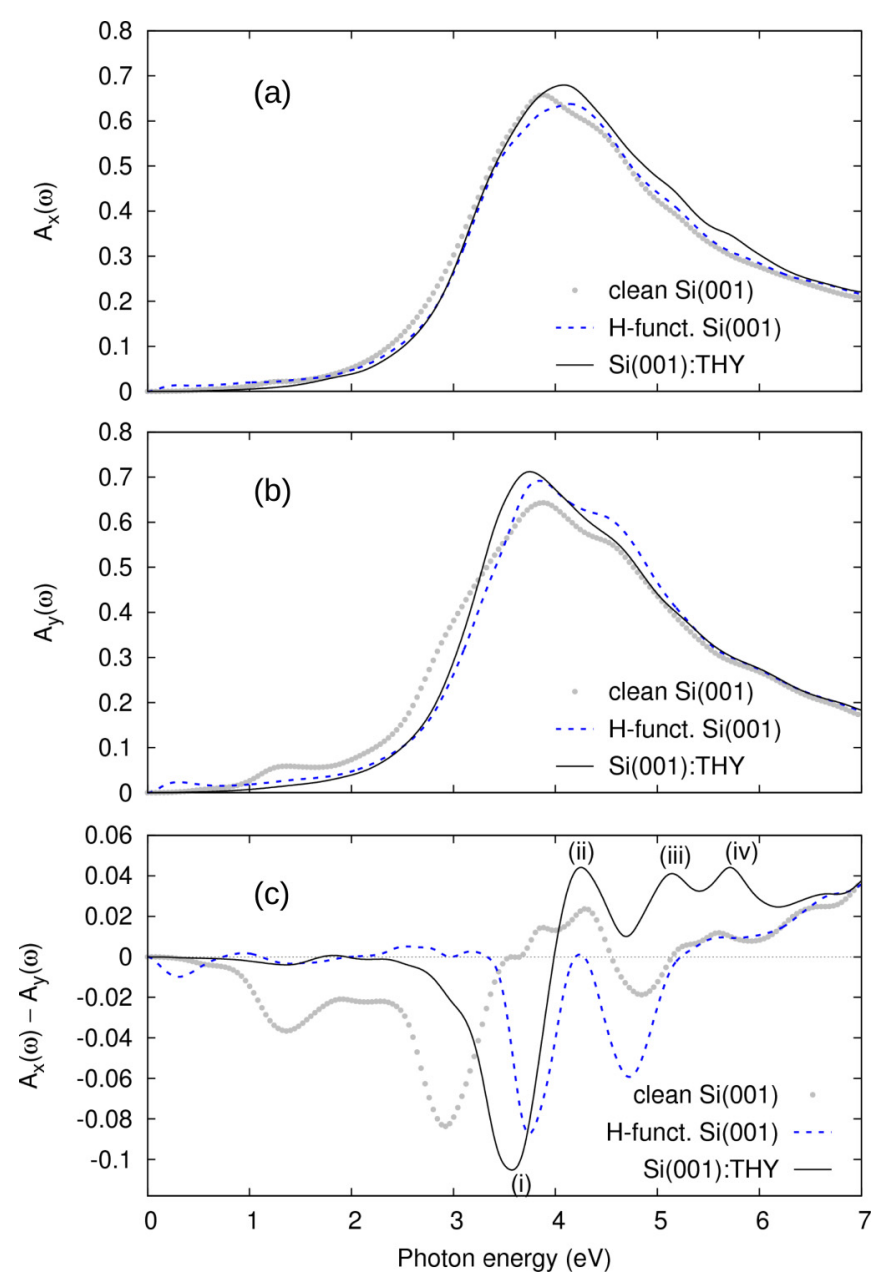

FIG. 4. Optical absorbance $A(\omega)$ for light polarization along the $x$ (a) and $y$ (b) directions, and their difference $\Delta A(\omega)=A_{x}(\omega)-$ $A_{y}(\omega)$ (c), for vertically adsorbed $\operatorname{Si}(001)$ :THY (black solid line) compared with that of clean $\operatorname{Si}(001)-c(4 \times 2)$ (gray circles). $A$ and $\Delta A$ are also reported for a model "H-functionalized" $\mathrm{Si}(001)$ surface without adsorbed nucleobases (short-dashed blue line, discussed in Sec. IV B).

systems given their similar RAS (Fig. 3). Consistently with the well-established RAS line shape of clean $\mathrm{Si}(001)-c(4 \times 2)$ (gray dots in Fig. 3), the absorbance of the reconstructed clean $\mathrm{Si}(001)$ slab shows a characteristic peak at $\sim 1.3 \mathrm{eV}$ and a shoulder feature at about $3 \mathrm{eV}$, only present for light polarized along the dimer rows [see $A_{y}$ in panel (b)]. Correspondingly, the absorption anisotropy $\Delta A=A_{x}-A_{y}$, which is reported in panel (c), clearly shows the two minima that correspond to the low-energy negative RAS spectrum of clean $\mathrm{Si}(001)$ (Fig. 3). Such features disappear in $\mathrm{Si}(001)$ :THY, which shows an almost flat $\Delta A$ up to about $3 \mathrm{eV}$. On the other hand, $\mathrm{Si}(001)$ :THY shows a significantly larger absorption for light polarized in the direction of the molecule rows $\left(A_{y}>A_{x}\right)$ in the region between 3.5 and $4 \mathrm{eV}$, whereas $A_{y} \approx A_{x}$ for clean $\mathrm{Si}(001)$ in the same energy range; this anisotropy corresponds to peak (i) in the RAS spectrum of Fig. 3. At higher energies three further peaks of $\Delta A$, having no correspondence in the clean $\mathrm{Si}(001)$ case, are present in the case of $\mathrm{Si}(001)$ :THY. The energy position of such peaks is roughly corresponding to that of peaks (ii), (iii), and (iv) in the RAS spectra, but, remarkably, with opposite sign. Consistently with the RAS presented above, the computed absorbance spectra of all the investigated systems are very similar to each other, sharing the main trends observed for vertical Si(001):THY (Fig. 4). We refer to the Supplemental Material for more details [43].

\section{DISCUSSION}

\section{A. Connection between RAS and absorbance spectra}

In order to understand the apparently puzzling sign of peaks (iii) and (iv), we point out the nontrivial connection between RAS and absorbance anisotropies, in terms of peak positions and even sign. This is evident when comparing the RAS spectrum of $\mathrm{Si}(001)$ :THY (black line in Fig 3) with the absorption anisotropy $\Delta A$ of the same system (black line in Fig. 4). The former is in fact characterized by four negative peaks, while the latter shows one negative peak, (i), and three positive ones, (ii)-(iv), although the energy positions are roughly corresponding. This is readily explained by noticing that while only the imaginary part of the dielectric tensor is needed for the absorbance as seen in Eq. (3), the RAS spectra computed by Eq. (2) also depend on the real part of $\varepsilon$. Indeed, one can rewrite $\Delta R / R$ as [30]

$$
\frac{\Delta R}{R}=\frac{4 \omega L}{c}\left[A_{\text {bulk }} \Delta \varepsilon^{\prime \prime}-B_{\text {bulk }} \Delta \varepsilon^{\prime}\right],
$$

where $\quad A_{\text {bulk }}=\left(\varepsilon_{\text {bulk }}^{\prime}-1\right) /\left[\left(1-\varepsilon_{\text {bulk }}^{\prime}\right)^{2}+\left(\varepsilon_{\text {bulk }}^{\prime \prime}\right)^{2}\right], \quad B_{\text {bulk }}=$ $\varepsilon_{\text {bulk }}^{\prime \prime} /\left[\left(1-\varepsilon_{\text {bulk }}^{\prime}\right)^{2}+\left(\varepsilon_{\text {bulk }}^{\prime \prime}\right)^{2}\right]$, and $\varepsilon^{\prime}$ and $\varepsilon^{\prime \prime}$ are for the real and imaginary parts, respectively. The RAS signal is hence affected by the frequency-dependent bulk dielectric function $\varepsilon_{\text {bulk }}$ (common to all cases considered here), through the functions $A_{\text {bulk }}(\omega)$ and $B_{\text {bulk }}(\omega)$, whose frequency dependence is shown in panel (b) of Fig. 5. Indeed, $A_{\text {bulk }}$ changes sign at $\hbar \omega \approx 3.83 \mathrm{eV}$, while $B_{\text {bulk }}$ is vanishingly small at low frequencies. Hence, RAS spectra contain contributions from both the first and the second term on the right-hand side of Eq. (4). Such contributions are singled out in Fig. 5(a) for $\mathrm{Si}(001)$ :THY. There one can see that the contribution by $\operatorname{Im}(\Delta \varepsilon)$ dominates at energies around $3.5 \mathrm{eV}$, corresponding to the anisotropy in the absorbance ( $A_{y}>A_{x}$ in Fig. 4), and is the main cause of RAS peak (i). Its precise energy position is slightly blueshifted because of growing participation by the $\operatorname{Re}(\Delta \varepsilon)$ term. Peak (ii), conversely, is mostly due to $\operatorname{Re}(\Delta \varepsilon)$ with the $\operatorname{Im}(\Delta \varepsilon)$ term suppressed by a small value of $A_{\text {bulk }}$. In this peak, as well as in the subsequent (iii) and (iv), the positive absorption anisotropy of Fig. 4 contributes to a negative RAS because of the sign of $A_{\text {bulk }}$. Overall, this analysis recalls that a direct interpretation of the RAS spectrum cannot be achieved by looking at the (polarized) absorbance only, since the full dielectric response of the substrate is even able to change the sign of the RAS peaks.

\section{B. Origin of the molecule-induced spectral features}

We now address the origin of the variations in the RAS and absorption spectra of $\mathrm{Si}(001)$ when uracil-like nucleobases are adsorbed. As shown by band structure results (Fig. 2), one notices that the energy difference between the molecular HOMO- and LUMO-related states of the $\mathrm{Si}(001): \mathrm{X}$ system 

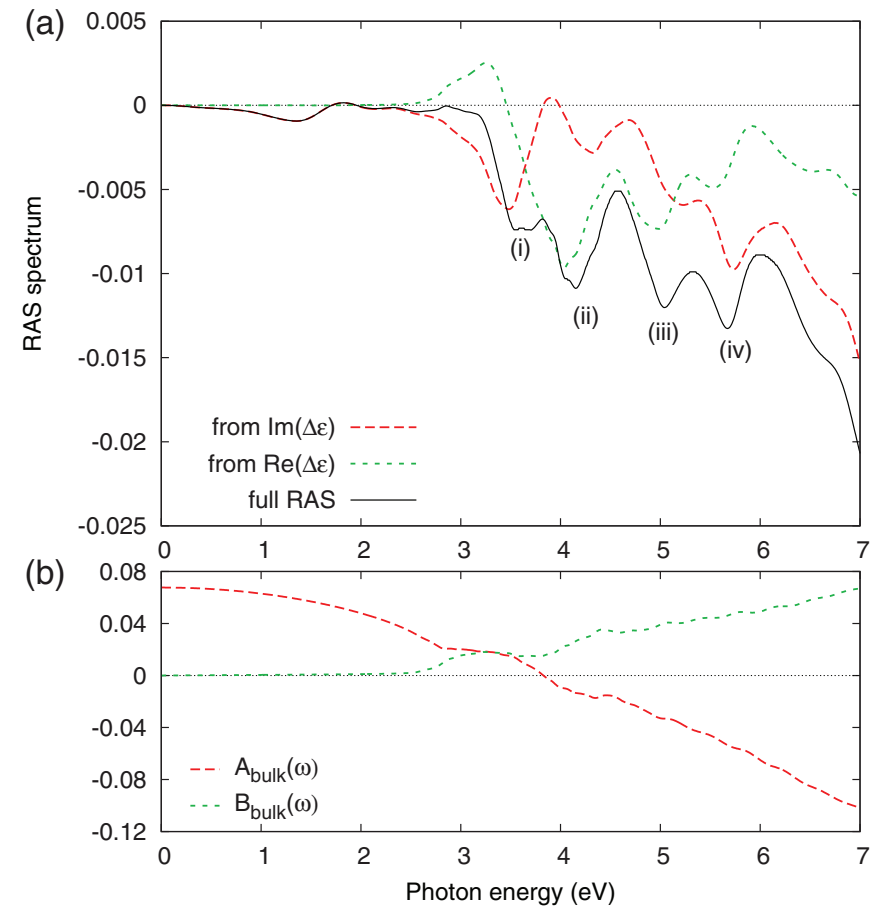

FIG. 5. (a) RAS spectrum of the $\mathrm{Si}(001)$ :THY system in vertical geometry (black solid line), showing contributions from $\operatorname{Im}(\Delta \varepsilon)$ and $\operatorname{Re}(\Delta \varepsilon)$ as a long-dashed red line and a short-dashed green one, respectively. (b) The functions $A_{\text {bulk }}(\omega)$ and $B_{\text {bulk }}(\omega)$; see Eq. (4).

amounts to about 3.5-4 eV, similar to the HOMO-LUMO gap of about $3.8 \mathrm{eV}$ we computed for gas phase thymine. This is the same energy window as the one where $A_{y}>A_{x}$ eventually producing peak (i) in panel (c) of Fig. 4. This may suggest attributing the origin of the latter to THY HOMO-LUMO transitions contributing to the optical absorption at this energy. However, dipole excitations by light polarized perpendicularly to the molecular plane (i.e., along the $y$ direction) are forbidden by symmetry, since both molecular states have $\pi$ character (see Fig. 2). As a further check we also computed the optical absorbance spectrum of a row of THY molecules. Practically, we keep the molecules in the same geometry they attain upon deposition on $\mathrm{Si}(001)$, but the latter being removed and the resulting dangling bonds suitably saturated (we make use of fictitious "half-H" atoms as numerical devices, as specified in Sec. S.B. of the Supplemental Material [43]). A negligible $y$ component of absorption with respect to the $x$ one is found in the whole analyzed energy range $(0-7 \mathrm{eV})$; in particular in the energy region of peak (i) only $A_{x}$ is detectable, at variance with the results on the full $\mathrm{Si}(001)$ :THY system where $A_{y}$ is strongly enhanced. This rules out a purely molecular explanation of the observed features. The same considerations hold for the absorption spectra of rows of uracil or 5-fluorouracil molecules [43].

The actual molecular contributions to the optical anisotropy can be highlighted by analyzing the spatial localization of the occupied and empty states involved in dipole-allowed transitions. In particular, we generalize Eq. (1) by weighting the valence and conduction states according to their localization $W_{v k}^{\Phi}=\sum_{\phi \in \Phi}\left|\left\langle\phi \mid \psi_{v k}\right\rangle\right|^{2}$ and $W_{c k}^{\Phi}=\sum_{\phi \in \Phi}\left|\left\langle\phi \mid \psi_{c k}\right\rangle\right|^{2}$, respectively, with $\Phi$ indicating either the set of Si (substrate)

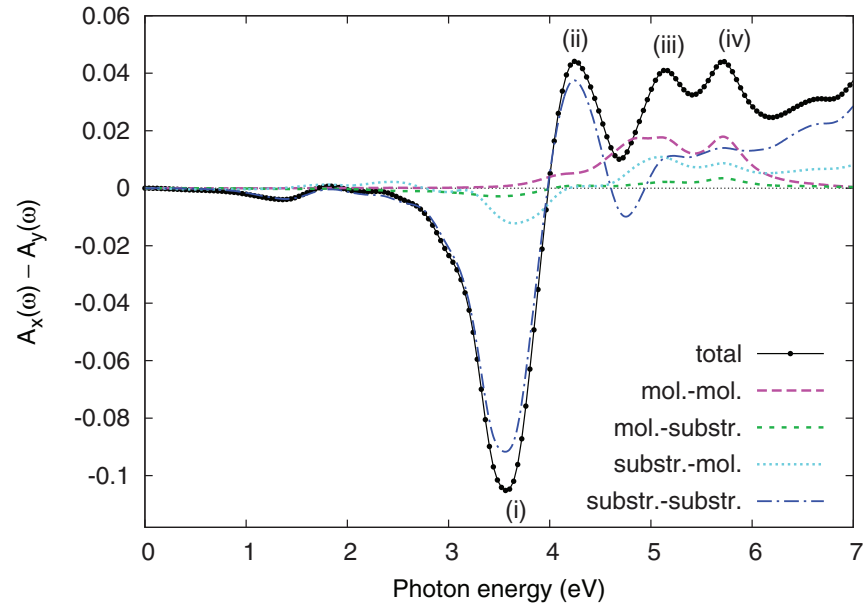

FIG. 6. Optical absorbance anisotropy, $\Delta A(\omega)=A_{x}(\omega)-$ $A_{y}(\omega)$, for the $\mathrm{Si}(001)$ :THY system with vertically adsorbed molecules (thick black line with dots). Thinner lines show the decomposition of $\Delta A(\omega)$ in terms of contributions due to transitions from valence to conduction states mostly localized on the substrate or on the THY molecule, as determined by Eq. (5).

or nucleobase (molecule) atoms and $\phi$ an atomic orbital belonging to one of these two parts [46]:

$$
\begin{aligned}
\operatorname{Im} \varepsilon_{j j}^{\Phi \Phi^{\prime}}(\omega)= & \frac{16 \pi}{\omega^{2}} \sum_{v, c, k} W_{v k}^{\Phi} W_{c k}^{\Phi^{\prime}} \\
& \times\left|\left\langle\psi_{v k}\left|(\mathbf{v})_{j}\right| \psi_{c k}\right\rangle\right|^{2} \delta\left(\epsilon_{c k}-\epsilon_{v k}-\omega\right) .
\end{aligned}
$$

We can hence disentangle four different contributions in the RAS spectra, according to the possible choices of $\Phi$ and $\Phi^{\prime}$. Such contributions are shown in Fig. 6, where peaks (i) and (ii) below $4.5 \mathrm{eV}$ clearly appear as being essentially due to states localized on the surface of the Si substrate (blue curve), even though they fall at the same energy of molecular HOMO-LUMO excitations. This is consistent with the already discussed independence of peaks (i) and (ii) on the adsorbed molecular species. Hence, the change in optical anisotropy up to $4.5 \mathrm{eV}$ shown in Fig. 3 can be ascribed to indirect effects that adsorption has on the substrate itself. Instead, a significant contribution from transitions among truly molecular states (magenta curve) appears in the $4.5-6 \mathrm{eV}$ range; see peaks (iii) and (iv). Consistently, the latter energy range is the one where RAS spectra show the largest sensitivity to the molecule type and geometry (Fig. 3). However, a non-negligible substrate contribution is also present in this energy window, due to the hybridization of molecular states with the Si substrate ones.

The indirect effects of molecular adsorption on the optical response can be further analyzed by recognizing two possible mechanisms: on the one hand, the geometry of the clean $\mathrm{Si}(001)$ dimers is altered upon adsorption of URA-like nucleobases in the "dimer bridge" configuration, with a slight elongation of Si dimers and a substantial reduction of their buckling, as already reported in our previous work [18]. On the other hand, surface dangling bonds are saturated by the adsorbed molecules (functionalization). To single out these contributions independently of the adsorbed species, we develop a model for a functionalized $\operatorname{Si}(001)$ surface that 
is intermediate between the clean $\mathrm{Si}(001)$ and $\mathrm{Si}(001)$ :THY cases. Namely, the model is obtained by removing the THY molecule, and placing "half- $\mathrm{H}$ " atoms in place of THY oxygens, in order to simulate the bare bond-saturation effect (we refer to the Supplemental Material for further details [43]). Si atoms are frozen in the geometry of the $\mathrm{Si}(001)$ :THY system, and hydrogen atoms cleaved from THY are left at their positions on alternating $\mathrm{Si}$ surface dimers. Figure 4 shows $A$ and $\Delta A$ of this $\mathrm{H}$-functionalized model (dashed blue line), to be compared with those of the clean $\mathrm{Si}(001)$ surface (gray dots) and those of $\mathrm{Si}(001)$ :THY (black line). One can notice the disappearance of the absorption anisotropy features characteristic of the clean $\mathrm{Si}(001)$ surface below $3.5 \mathrm{eV}$ [Fig. 4(c)]. The prevalent absorption of $\mathrm{Si}(001)$ :THY along the $y$ direction in the $3.5-4 \mathrm{eV}$ range is also well reproduced by the $\mathrm{H}$-functionalized model, although at a slightly higher energy. This again demonstrates that substrate modifications induced by molecular adsorption, giving rise to the flattening of the RAS spectrum and to the appearance of peaks (i) and (ii) in Fig. 3, are completely independent of the adsorbed species, consistently with the observations made above.

Further analysis with an additional model where the clean surface is deformed to the same coordinates as in $\mathrm{Si}(001)$ :THY, but with no adsorbed molecule nor hydrogen, shows that the flattening of the RAS at low energies is already almost complete with structural modifications only, whereas bond saturation is necessary in order to attain the anisotropy producing a prominent peak (i) [43]. On the contrary, the higher energy molecular contributions giving rise to the positive $\Delta A$ anisotropy [peaks (iii) and (iv)] observed for the Si(001):THY system (Fig. 6) are not reproduced by any of these intermediate models, consistently with the substantial molecular character of the involved transitions.

\section{Chemical sensitivity of RAS spectra}

In order to understand the chemical sensitivity appearing in the near-UV spectral range [in particular the energy variations of RAS peak (iv) in Fig. 3] we consider again the case of unsupported rows of molecules, i.e., in the absence of the substrate and saturated by the "half-H" atoms [43], and we analyze the orbital contributions to the optical response. To facilitate comparison to the adsorbed case, we evaluate the absorption anisotropy through Eq. (3) and report the resulting $\Delta A$ spectra in Fig. 7 for THY, URA, and 5-FU. (These spectra are actually dominated by $A_{x}$, with $A_{y} \approx 0$; see Fig. S3 of the Supplemental Material [43].) Two main structures are identified and marked in the figure by vertical lines, presenting a distinctive and opposite dependence in going from URA to THY to 5-FU. However, the contribution by the lower energy feature $(\approx 4 \mathrm{eV})$ to the RAS of the corresponding $\mathrm{Si}: \mathrm{X}$ systems is suppressed by a small value of $A_{\text {bulk }}$ in Eq. (4) and overwhelmed by substrate terms, whereas that at about $5.5 \mathrm{eV}$ can be clearly associated with peak (iv) in the RAS, displaying the same nucleobase dependence, with shifts with respect to THY of $-0.12 \mathrm{eV}$ for URA and $+0.11 \mathrm{eV}$ for 5 -FU.

The observed chemical dependence of $\Delta A$ for the molecular rows can be fully rationalized in terms of the molecular orbitals involved in the corresponding transitions, and helps understanding the sensitivity of the RAS on the specific
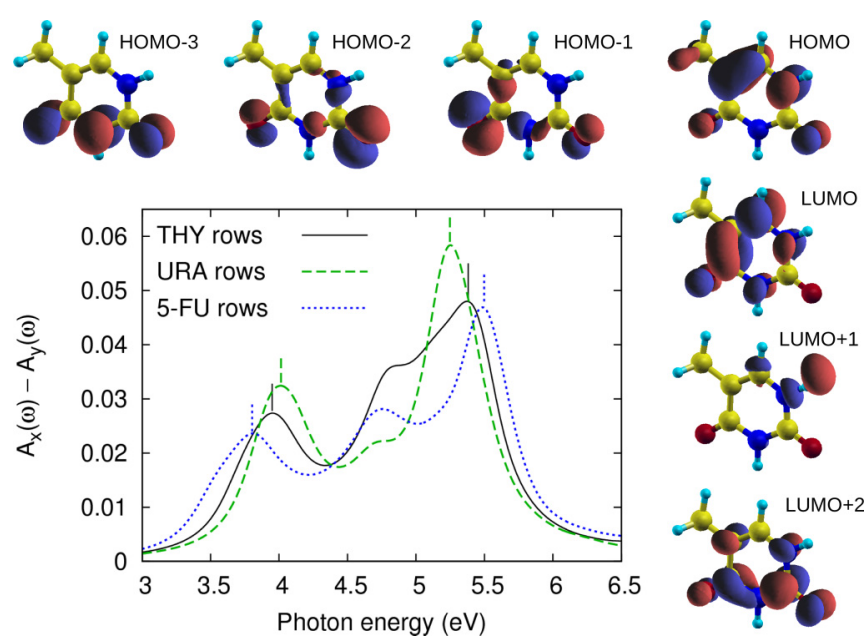

FIG. 7. Center panel: Optical absorbance anisotropy, $\Delta A(\omega)=$ $A_{x}(\omega)-A_{y}(\omega)$, for freestanding molecular rows (see text): THY (black solid line), URA (green long-dashed line), and 5-FU (blue dots). Vertical lines mark the energy positions of the two main peaks of each system, clearly displaying an opposite trend in going from URA to THY to 5-FU, consistently with the energy position of frontier orbitals (see text). Surrounding panels show molecular orbitals of gas phase thymine.

nucleobase. We remark that the three molecules have similar frontier molecular orbitals, whose relative energy is affected by chemical functionalization and especially by adsorption as well as by band dispersion in the molecular rows (see the evolution of $\pi$ and $\sigma$ character of the states along the SBZ in Fig. S3 of the Supplemental Material [43]). To avoid ambiguities, we choose as a reference for the subsequent analysis the case of gas phase THY, whose orbitals are reported in Fig. 7: we then add the ${ }^{(g)}$ superscript to denote the states having the same symmetry as those depicted therein. A detailed analysis of the molecular orbitals for the rows shows that the feature at lower energy is mostly due to transitions between $\mathrm{HOMO}^{(\mathrm{g})}$ and $\mathrm{LUMO}^{(\mathrm{g})}$ states. The most prominent feature, at about $5.5 \mathrm{eV}$, is instead due to transitions between $\mathrm{HOMO}-3^{(\mathrm{g})}$ and $\mathrm{LUMO}+2^{(\mathrm{g})}$ states. Other features at intermediate energies involve transitions between states with the same symmetry as the $\mathrm{HOMO}-3^{(\mathrm{g})} \rightarrow \mathrm{LUMO}^{(\mathrm{g})}$ and $\mathrm{HOMO}^{(\mathrm{g})} \rightarrow \mathrm{LUMO}+2^{(\mathrm{g})}$, whereas states with $\sigma$ symmetry are practically invisible. Returning to the nucleobase dependence of the two main features in Fig. 7, the $\mathrm{HOMO}^{(\mathrm{g})} \rightarrow$ LUMO $^{(\mathrm{g})}$ energy $(\approx 4 \mathrm{eV})$ lowers from URA to THY to 5-FU, consistently with the experimental findings for the HOMO $\rightarrow$ LUMO transition of these molecules in solution [47]. There, this difference was ascribed to a destabilizing effect induced by the functional group on the HOMO state, where increasingly strong antibonding contributions are found for THY and 5-FU with respect to URA, hence the lower excitation energy; conversely, the LUMO state is less affected by changes in the functional group bound at position 5 , due to the lower wave function amplitude on that part of the molecule (see Fig. 7). The same explanation holds for the opposite trend of the higher energy feature, and hence the (iv) peak in the RAS: there, the LUMO $+2^{(\mathrm{g})}$ states are destabilized in passing from URA to THY to 5-FU, whereas the HOMO $-3^{(\mathrm{g})}$ states are unaffected having largest amplitude at the opposite part of 
the molecules. On the basis of this analysis such shifts are not expected for HOMO $-3^{(\mathrm{g})} \rightarrow \mathrm{LUMO}^{(\mathrm{g})}$ (both unaltered) and $\mathrm{HOMO}^{(\mathrm{g})} \rightarrow \mathrm{LUMO}+2^{(\mathrm{g})}$ (both destabilized) transitions, nor are they observed in the $\Delta A$ values reported in Fig. 7.

\section{CONCLUSIONS}

We calculated the optical properties, including reflectance anisotropy spectra (RAS), for the $\mathrm{Si}(001)$ surface covered with thymine, uracil, and 5-fluorouracil molecules in the "dimer bridge" configuration, also taking into account a possible tilting of the adsorbed molecules with respect to the surface normal.

We determine clearly distinguishable effects occurring upon molecular adsorption, and how they influence absorbance and reflectance anisotropy spectra. On one side, the strong attenuation and flattening of the typical absorption anisotropy and RAS features of the clean surface in the low-energy part of the spectra (up to $3 \mathrm{eV}$ ), mostly due to a deformation of $\mathrm{Si}$ dimer geometry, is accompanied by the appearance of a characteristic negative peak in absorption anisotropy in the $3.5-4 \mathrm{eV}$ range (resulting in negative features in the RAS), that we may describe as a "Si bond saturation effect". The latter is mostly due to a change in the chemistry (coordination) of the surface $\mathrm{Si}$ atoms. Both these effects are independent of molecule type (thymine, uracil, 5-fluorouracil) and tilting, resulting in almost indistinguishable RAS spectra for the different investigated adsorbed nucleobases up to $\sim 4 \mathrm{eV}$.

Molecular transitions, although possibly starting in the same energy range, are shown to contribute only above $4 \mathrm{eV}$ (near UV region). There, the spectra consequently show a moderate dependence on the specific adsorbed molecule, through the influence of chemical substitutions on orbital energies, and on its tilt angle. The above picture is confirmed by a decomposition of electronic transitions, based on the spatial localization of the corresponding electronic states and by explicitly evaluating the effects of structural and bonding properties of Si surface atoms through suitable models where the adsorbed molecules or the substrate are eliminated and replaced by an artificial saturation of dangling bonds.

The combination of RAS spectroscopy measurements with $a b$ initio calculations represents a suitable and effective tool to characterize these and similar organically functionalized silicon surfaces, by allowing one to detect specific spectral features related either to the type of molecule-surface bonding (e.g., the "dimer bridge" configuration), or to the chemical and structural details of the absorbed molecules.

\section{ACKNOWLEDGMENTS}

E.M. acknowledges financial support from Regione Autonoma della Sardegna under Project R.A.S. L. R. 07/08/2007 CRP-26666, and from Fondazione di Sardegna under Project UniCa "PRID 2015". G.C. acknowledges financial support from IDEA-AISBL, Bruxelles, Belgium. The authors acknowledge the CINECA award under the ISCRA initiative, for the availability of high-performance computing resources and support (Project HP10CDF3LP: "OptNclSi: Optical properties of uracil-like nucleobases adsorbed on silicon(001)"). G.O. acknowledges ETSF-Italy for computational resources [48]. We thank Nicola Manini for a careful reading of the manuscript.
[1] D. Branton, D. W. Deamer, A. Marziali, H. Bayley, S. A. Benner, T. Butler, M. Di Ventra, S. Garaj, A. Hibbs, X. Huang, S. B. Jovanovich, P. S. Krstic, S. Lindsay, X. S. Ling, C. H. Mastrangelo, A. Meller, J. S. Oliver, Y. V. Pershin, J. M. Ramsey, R. Riehn, G. V. Soni, V. Tabard-Cossa, M. Wanunu, M. Wiggin, and J. A. Schloss, Nat. Biotechnol. 26, 1146 (2008).

[2] D. Fologea, M. Gershow, B. Ledden, D. S. McNabb, J. A. Golovchenko, and J. Li, Nano Lett. 5, 1905 (2005).

[3] A. Vilan, O. Yaffe, A. Biller, A. Salomon, A. Kahn, and D. Cahen, Adv. Mater. 22, 140 (2010).

[4] R. A. Wolkow, Annu. Rev. Phys. Chem. 50, 413 (1999).

[5] A. M. Paraian, U. Rossow, S. Park, G. Salvan, M. Friedrich, T. U. Kampen, and D. R. T. Zahn, J. Vac. Sci. Technol. B 19, 1658 (2001)

[6] L. Beverina and P. Salice, Eur. J. Org. Chem. 2010, 1207 (2010).

[7] G. Cappellini, G. Malloci, and G. Mulas, Superlattices Microstruct. 46, 14 (2009).

[8] R. Cardia, G. Malloci, A. Mattoni, and G. Cappellini, J. Phys. Chem. A 118, 5170 (2014).

[9] D. Costa, C.-M. Pradier, F. Tielens, and L. Savio, Surf. Sci. Rep. 70, 449 (2015).

[10] T. Yamada, K. Shirasaka, A. Takano, and M. Kawai, Surf. Sci. 561, 233 (2004).

[11] F. Allegretti, M. Polcik, and D. P. Woodruff, Surf. Sci. 601, 3611 (2007).
[12] S. Irrera and N. H. De Leeuw, Proc. R. Soc. A 467, 1959 (2011),

[13] S. D. Silaghi and D. R. T. Zahn, Appl. Surf. Sci. 252, 5462 (2006).

[14] C. I. Smith, A. Bowfield, G. J. Dolan, M. C. Cuquerella, C. P. Mansley, D. G. Fernig, C. Edwards, and P. Weightman, J. Chem. Phys. 130, 044702 (2009).

[15] K. Seino, W. G. Schmidt, M. Preuss, and F. Bechstedt, J. Phys. Chem. B 107, 5031 (2003).

[16] K. Seino, W. G. Schmidt, and F. Bechstedt, Phys. Rev. B 69 , 245309 (2004).

[17] A. Lopez, Q. Chen, and N. V. Richardson, Surf. Interface Anal. 33, 441 (2002).

[18] E. Molteni, G. Onida, and G. Cappellini, Eur. Phys. J. B 89, 98 (2016).

[19] P. Hohenberg and W. Kohn, Phys. Rev. 136, B864 (1964).

[20] W. Kohn and L. J. Sham, Phys. Rev. 140, A1133 (1965).

[21] J. P. Perdew, K. Burke, and M. Ernzerhof, Phys. Rev. Lett. 77, 3865 (1996).

[22] GGA-PBE was verified to produce qualitatively equivalent results as for band structure, geometry optimization, and optical absorption to the local density approximation (LDA) adopted in Ref. [18].

[23] M. Fuchs and M. Scheffler, Comput. Phys. Commun. 119, 67 (1999).

[24] H. J. Monkhorst and J. D. Pack, Phys. Rev. B 13, 5188 (1976). 
[25] G. Onida, L. Reining, and A. Rubio, Rev. Mod. Phys. 74, 601 (2002).

[26] K. Seino and W. G. Schmidt, Surf. Sci. 548, 183 (2004).

[27] G. Onida, R. Del Sole, M. Palummo, O. Pulci, and L. Reining, Phys. Status Solidi A 170, 365 (1998).

[28] C. Hogan, R. Del Sole, and G. Onida, Phys. Rev. B 68, 035405 (2003).

[29] K. Gaal-Nagy and G. Onida, Phys. Rev. B 75, 155331 (2007).

[30] C. Hogan and R. Del Sole, Phys. Status Solidi B 242, 3040 (2005).

[31] F. Manghi, R. Del Sole, A. Selloni, and E. Molinari, Phys. Rev. B 41, 9935 (1990).

[32] F. Bechstedt, L. Matthes, P. Gori, and O. Pulci, Appl. Phys. Lett. 100, 261906 (2012).

[33] B. Adolph, V. I. Gavrilenko, K. Tenelsen, F. Bechstedt, and R. Del Sole, Phys. Rev. B 53, 9797 (1996).

[34] L. Yang, J. Deslippe, C.-H. Park, M. L. Cohen, and S. G. Louie, Phys. Rev. Lett. 103, 186802 (2009).

[35] P. Giannozzi, S. Baroni, N. Bonini, M. Calandra, R. Car, C. Cavazzoni, D. Ceresoli, G. L. Chiarotti, M. Cococcioni, I. Dabo, A. Dal Corso, S. de Gironcoli, S. Fabris, G. Fratesi, R. Gebauer, U. Gerstmann, C. Gougoussis, A. Kokalj, M. Lazzeri, L. Martin-Samos, N. Marzari, F. Mauri, R. Mazzarello, S. Paolini, A. Pasquarello, L. Paulatto, C. Sbraccia, S. Scandolo, G. Sclauzero, A. P. Seitsonen, A. Smogunov, P. Umari, and R. M. Wentzcovitch, J. Phys. Condens. Matter 21, 395502 (2009).

[36] A. Marini, C. Hogan, M. Gruening, and D. Varsano, Comput. Phys. Commun. 180, 1392 (2009).

[37] A. Ravikumar, A. Baby, H. Lin, G. P. Brivio, and G. Fratesi, Sci. Rep. 6, 24603 (2016).
[38] M. Palummo, G. Onida, R. Del Sole, and B. S. Mendoza, Phys. Rev. B 60, 2522 (1999).

[39] L. Caramella, C. Hogan, G. Onida, and R. Del Sole, Phys. Rev. B 79, 155447 (2009).

[40] M. Palummo, N. Witkowski, O. Pluchery, R. Del Sole, and Y. Borensztein, Phys. Rev. B 79, 035327 (2009).

[41] R. Shioda and J. van der Weide, Phys. Rev. B 57, R6823(R) (1998).

[42] P. Weightman, D. S. Martin, R. J. Cole, and T. Farrell, Rep. Prog. Phys. 68, 1251 (2005).

[43] See Supplemental Material at http://link.aps.org/supplemental/ 10.1103/PhysRevB.95.075437 for absorbance spectra of $\mathrm{Si}(001)$ :URA, Si(001):5-FU, and $\mathrm{Si}(001)$ :THY tilted 20 ; absorbance spectra and band structures of THY, URA, and 5-FU rows in the same geometry as in the corresponding vertical $\mathrm{Si}(001)$ :X systems; ball and stick model of the distorted $\mathrm{Si}(001)$ surface functionalized by $\mathrm{H}$ atoms; absorbance anisotropy of a model $\mathrm{Si}(001)$ surface with $\mathrm{Si}$ atoms frozen in the geometry of the $\mathrm{Si}(001)$ :THY system, but without any functionalization; specification of the "half-H" potential, including Refs. [44,45].

[44] L.-W. Wang and J. Li, Phys. Rev. B 69, 153302 (2004).

[45] S. Iacobucci, P. Alippi, P. Calvani, M. Girolami, F. Offi, L. Petaccia, and D. M. Trucchi, Phys. Rev. B 94, 045307 (2016).

[46] E. Cinquanta, G. Fratesi, S. dal Conte, C. Grazianetti, F. Scotognella, S. Stagira, C. Vozzi, G. Onida, and A. Molle, Phys. Rev. B 92, 165427 (2015).

[47] T. Gustavsson, N. Sarkar, A. Bányász, D. Markovitsi, and R. Improta, Photochem. Photobiol. 83, 595 (2007).

[48] A. Matsuura, N. Thrupp, X. Gonze, Y. Pouillon, G. Bruant, and G. Onida, Comput. Sci. Eng. 14, 22 (2012). 